\title{
Determinación de variables fenotípicas, sus interrelaciones y componentes principales en hembras de un hato ovino
}

\section{Phenotypic variables determination, interrelationship and principal components in ovine herd females}

\author{
Marlon Hernández Baca ${ }^{1}$ Guadalupe Centeno Martínez ${ }^{2}$ \\ ${ }^{1}$ MSc. en sistemas integrales de producción animal en el trópico/ ORCID: https://orcid.org/0000-0002-5488-8282 \\ ${ }^{2}$ Ingeniera zootecnista. \\ Universidad Nacional Agraria
}

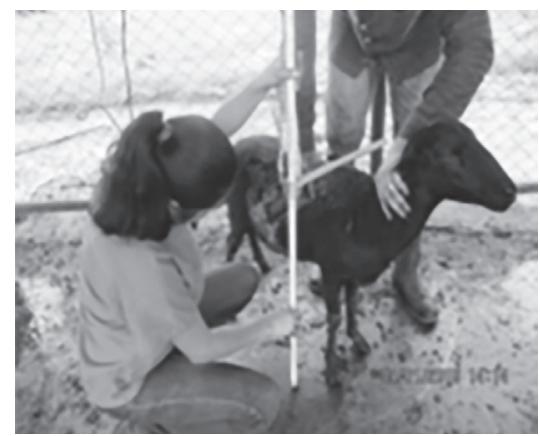

\section{RESUMEN}

El hato ovino presente en el país, por encontrarse mayormente en economías rurales de bajos ingresos, no ha tenido la importancia apropiada para el desarrollo de estudios que permitan realizar una adecuada caracterización del mismo. El presente estudio, pretende dar un paso adelante en ese sentido al tener como objetivo la valoración faneróptica y la determinación de variables morfométricas, sus interrelaciones y componentes principales (ACP) de la explicación de la varianza. Se evaluaron un total de 31 hembras por muestreo aleatorio, pertenecientes a un hato de 100 individuos de la finca Santa Rosa, en la comunidad de Sabana Grande, departamento de Managua. Se determinaron 14 medidas morfológicas, siete caracteres fanerópticos, y se estimaron nueve índices zoométricos, de los cuales cinco fueron etnológicos: Cefálico (IC), dáctilo torácico (IDT), corporal (ICo), proporcionalidad (IPRO), pelviano (IPE); y cuatro de capacidad productiva: Profundidad relativa del tórax (IPRT), pelviano transversal (IPET), pelviano longitudinal (IPEL), y espesor relativo de la caña (IERC). Por estadística descriptiva se determinó que un $46.67 \%$ de las variables morfológicas presento una variabilidad media, un $33.33 \%$ una variabilidad baja y un $20 \%$ una variabilidad alta, la menor variabilidad fue para (ALC) con un CV de $3.65 \%$ y la de mayor variación correspondió (LG) con un CV de $16.76 \%$, reflejando que los individuos no presentan una alta variabilidad morfoestructural. La mayor parte de las correlaciones entre las variables estudiadas resultaron positivas, con excepción de largo de la grupa (LG) que se correlaciono negativamente con (PV) y (AP), y el perímetro de la caña $(\mathrm{PC})$ que se correlaciono negativamente con todas las variables estudiadas. La mayoría de las correlaciones resultaron significativas, con excepción de la correlación entre (AP)

\section{ABSTRACT}

The sheep herd present in the country, because it is mostly found in low-income rural economies, has not been of appropriate importance for studies development that permit an adequate characterization of it. The present study, intends to take a step forward in that sense, by having as objective the phaneroptic valuation and the morphometric variables determination, interrelationship and main components (ACP) of the variance explanation. Thirty one females were evaluated by random sampling, belonging to 100 animals herd, from the Santa Rosa farm, in the Sabana Grande community, of Managua department. Fourteen morphological measurements were determined, seven phaneroptic characters, and nine zoomometric indexes were estimated, five were ethnological: Cephalic (IC), thoracic dactyl (IDT), corporal (ICo), proportionality (IPRO), pelvic (IPE); and four to productive capacity: relative depth of the thorax (IPRT), transverse pelvic (IPET), longitudinal pelvic (IPEL), and relative thickness of the cane (IERC). By descriptive statistics it was determined that $46.67 \%$ of the morphological variables presented an medium variability, $33.33 \%$ a low variability and $20 \%$ a high variability, the lowest variability was for (ALC) with a CV of $3.65 \%$ and the highest variation corresponded (LG) with a $\mathrm{CV}$ of $16.76 \%$, reflecting that individuals do not present a high morphostructural variability. The Most correlations among the variables studied were positive, except length of the rump (LG) that was negatively correlated with (PV), (AP), and perimeter of cane (PC) that correlated negatively with all the variables studied. All correlations were significant, exception the correlation between (AP) and (AC), and (LG) with (PC) whose correlations were not significant respect to all other variables. Based on the zoometric indexes, these sheep can be defined as, dolichocephal-
Recibido: 25 de enero del 2019

Aceptado: 23 de julio del 2019

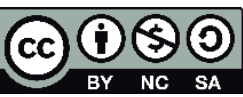

C Copyright 2019. Universidad Nacional Agraria
Los artículos de la revista La Calera de la Universidad Nacional Agraria, Nicaragua, se comparten bajo términos de la licencia Creative Commons: Reconocimiento, No Comercial, Compartir Igual. Las autorizaciones adicionales a las aquí delimitadas se pueden obtener en el correo edgardo.jimenez@ci.una.edu.ni 
y (AC), y (LG) con (PC) cuyas correlaciones no resultaron significativas respecto a todas las demás variables. En base a los índices zoométricos estas ovejas pueden definirse como, dolicocéfalas, hipermétricas, brevilíneas, mediolíneas y convexilíneas, por otro lado los índices de proporcionalidad (IPRO), profundidad relativa del tórax (IPRT) y el pelviano transversal (IPET), revelaron que los animales del hato no presentan un formato con clara definición a la actitud cárnica y en relación a la faneróptica, se trata de ovejas con capa de color café y negras, mucosas negras, pezuñas oscuras, ubres pigmentadas, grupas rectas, perfiles ligeramente convexos y orejas horizontales hacia los lados. Con el análisis multivariado de ACP, se determinó que las variables funcionales más destacadas son LC, AC, PV, LG, PT y DE, las que se pueden tener en cuenta para procesos de selección del hato actual.

Palabras clave: morfometría, faneróptica, zoometría, correlaciones. ic, hypermetric, brevilineal, medialineal and convexilineal, on the other hand the proportionality indexes (IPRO), relative depth of the thorax (IPRT) and the transverse pelvic (IPET), revealed that these animals, don't have format clear to meat attitude, In relationship to faneroptical characteristics, these are sheep, with brown and black coat, black mucous, dark hooves, pigmented udders, straight rumps, slightly convex profiles and horizontal ears towards the sides. With the multivariate analysis of ACP, it was determined that the most important functional variables were, LC, AC, PV, LG, PT and DE, which can be taken into account for selection processes of the current herd.

Keywords: morphometry, phaneroptic, zoometry, correlations.

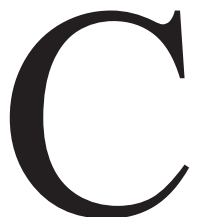

olón en su segundo viaje introduce los ovinos a América. Según Rodero y cols (citado en Delgado y Nogales, 2009), los primeros ovinos llegados a América, fueron tomados de la Gomera (Islas Canarias), y trasladados a la isla La Española, hoy Santo Domingo, en 1493. Estos ovinos no seguían ningún criterio de raza, es decir, eran animales de diversa procedencia genética. A finales del mismo año, parte de estos animales, fueron transferidos a la isla Isabela, hoy isla de Cuba, según, Minola (citado por Delgado y Nogales, 2009). Posteriormente se difundieron rápidamente siendo los países de mayores censos Guatemala, México, Nicaragua, Bolivia, Colombia, Ecuador y Perú.

Los ovinos llegados a Nicaragua, pertenecían a dos agrupaciones raciales, referidas a ovejas de lana basta y larga, y a otro grupo de animales carentes de lana y con fibras pilosas (Delgado y Nogales, 2009), los cuales se asentaron principalmente en la zona del pacifico y central del país, en correspondencia con los principales asentamientos humanos establecidos por los conquistadores. Ya para el siglo XVI, los ejemplares ovinos recibidos correspondían a los grupos raciales, Merino y Canaria, que correspondían a los explotados en la zona sur de la Península Ibérica y las Islas Canarias.

Hasta los años 90, no existía una referencia puntual del número de ovinos y su distribución en el territorio nacional, realmente se especulaba con cifras que rondaban las 10000 cabezas. Sin embargo, esto cambio con la llegada de los resultados del tercer censo nacional agropecuario, en la cual la cifra poblacional asciende a 29915 cabezas.
En la región del pacifico se localiza el $65.90 \%$ del hato ovino, siendo Managua el departamento de mayor población nacional, con un $14.68 \%$, seguido por los departamentos de León $9.63 \%$ y Masaya $9.56 \%$. Estos tres departamentos constituyen el denominado triángulo ovejero y en conjunto representan el $33.89 \%$ del hato nacional ovino, destacando también los departamentos de Rivas, Carazo, y Matagalpa en la región central del país.

En relación a las razas, en los años 70, del siglo pasado, de manera aislada y exótica aún se observaban algunos ovinos lanudos criollos, con funciones más ornamentales que productivas. Iniciándose el auge ovino en los 80 con la importación de Cuba de ovejas Pelibuey, según la organización para la alimentación y la agricultura (FAO, 2005), a la que posteriormente se agregaron las razas, Black belly, Kathadin y más recientemente las razas Dorper, Suffolk y Texel, todas ellas de orientación cárnica.

Muy pocos estudios en el país se han desarrollado de manera científica, para caracterizar la zoometría de estos animales, lo cual es de gran relevancia para conocer los principales indicadores morfológicos, que bajo nuestras condiciones son importantes tener en cuenta para seleccionar adecuadamente los pies de crías y desarrollar programas de mejora en dicha especie. Y es lo que pretendemos con nuestro estudio, aportando elementos que permitan una mejor caracterización fenotípica y una mejor valoración productiva de los individuos, dejando sentada una base metodológica para el estudio morfológico y zoométrico de los ovinos en el país. 


\section{MATERIALES Y MÉTODOS}

El presente estudio se desarrolló en la finca Santa Rosa, en la comunidad de Sabana Grande, departamento de Managua, ubicada en las coordenadas $12^{\circ} 08^{\prime} 15.53^{\prime \prime}$ latitud norte, y $86^{\circ} 09^{\prime} 58.84^{\prime \prime}$ longitud oeste, con una elevación de $68 \mathrm{msnm}$; con clima seco tropical de sabana, precipitaciones entre 190 y $1395 \mathrm{~mm}$, temperaturas de 22 a $36^{\circ} \mathrm{C}$, humedad relativa mayor al $68 \%$, y vientos con velocidades de $10 \mathrm{~m} \mathrm{seg}^{-1}$. Instituto Nicaragüense de Estudios Territoriales (INETER, 2015).

De manera preliminar, se tomó una muestra de la variable peso vivo en 15 individuos del hato, se utilizó la media y la desviación estándar de dicha variable, junto con el tamaño conocido del hato y un error muestral considerado de 0.025 , para estimar el tamaño de muestra por la formula señalada por Cruz (2005), para poblaciones finitas de animales de granja.

Se tomaron 14 medidas Zoométricas, teniendo en cuenta la metodología descrita por Herrera et al. (citado por Bravo y Sepúlveda, 2010), diferenciadas en 4 cefálicas y 10 del tronco y extremidades, a la primera correspondieron, Ancho de la cabeza (AC), Largo de la cabeza (LC), Longitud de la cara (LCA) y Longitud del cráneo (LCR); y para la siguiente se determinaron, Alzada a la cruz (ALC), Alzada a la grupa (ALG), Diámetro longitudinal (DL), Ancho de pecho (AP), Ancho de grupa anterior (AG), Largo de la grupa (LG), Diámetro dorso-esternal (DE), Perímetro de la caña (PC), Perímetro torácico (PT) y Peso vivo del animal (PV). De estas medidas corporales se derivaron 9 índices zoométricos, 5 Índices etnológicos: Índice Cefálico (IC), Índice Dáctilo Torácico (IDT), Índice Corporal (ICo), Índice de proporcionalidad (IPRO), Índice Pelviano (IPE); y 4 índices de capacidad productiva (capacidad cárnica): Índice de Profundidad relativa del tórax (IPRT), Índice Pelviano transversal (IPET), Índice Pelviano longitudinal (IPEL), e Índice del espesor relativo de la caña (IERC). Por cada animal se observaron y se registraron las características fanerópticas relacionadas con la pigmentación de la mucosa, pezuñas y ubres, así como características morfológicas de tamaño de las orejas, dirección de las orejas, perfil cefálico, e inclinación de la grupa.

El procesamiento estadístico de la información se realizó utilizando el software Excel, en el que se consolido la base de datos con las medidas corporales y estimación de los índices morfológicos. Posteriormente dichas variables se procesaron con el software MINITAB 17, para obtener valores de tendencia central y de correlaciones entre ellas. De la matriz de correlaciones se derivó un análisis de componentes principales (ACP), con el fin de emplear las mismas escalas en todas las medidas y determinar el número de variables cuantitativas que recogen la mayor parte de la variación en los caracteres morfológicos estudiados.

\section{RESULTADOS Y DISCUSIÓN}

Características morfométricas de las ovejas en estudio. Las ovejas estudiadas, con una edad promedio de 1.86 años, presentan un alto mestizaje no registrado, entre las razas Pelibuey, Black Belly, Kathadin y Dorper negro, encontrando que en su morfometría las variables con mayor variación morfoestructural (CV mayor del 10\%) fueron, largo de la grupa con un $\mathrm{CV}$ de $16.76 \%$ y PV con $12.20 \%$, las demás fluctuaron en un rango de $3.65 \%$ a $9.49 \%$, que según Herrera et al. (como se citó en Salamanca, 2016), se ubicarían entre escasa variabilidad (inferiores al $4 \%$ ) y de variabilidad media (entre 5\% y 9\%). En el cuadro 1, se detallan los valores de las variables morfométricas y el peso vivo.

Cuadro 1. Diferentes medidas corporales en las ovejas estudiadas. $\mathrm{n}=31$

\begin{tabular}{crrrrr}
\hline Variable & Media & St dev & Cof var & Mínimo & Máximo \\
\hline PV & 32.37 & 3.95 & 12.20 & 26.40 & 39.30 \\
AC & 9.93 & 0.94 & 9.49 & 6.30 & 10.70 \\
LC & 23.27 & 1.08 & 4.66 & 21.37 & 25.80 \\
LCA & 15.08 & 1.35 & 4.56 & 14.26 & 16.97 \\
LCR & 8.20 & 0.69 & 8.48 & 6.53 & 8.83 \\
ALC & 63.53 & 2.32 & 3.65 & 58.60 & 68.00 \\
ALG & 62.54 & 2.37 & 3.79 & 57.50 & 67.00 \\
DL & 63.53 & 5.11 & 8.05 & 55.00 & 76.00 \\
AP & 16.00 & 1.06 & 6.64 & 14.30 & 18.50 \\
AG & 15.79 & 0.92 & 5.80 & 13.50 & 17.40 \\
LG & 20.30 & 3.40 & 16.76 & 15.00 & 33.00 \\
DE & 26.18 & 1.71 & 6.56 & 21.80 & 30.10 \\
PC & 12.11 & 0.81 & 6.74 & 11.00 & 14.00 \\
PT & 83.57 & 4.03 & 4.83 & 73.00 & 88.00 \\
\hline
\end{tabular}

Peso vivo. Se encontró un valor medio de $32.37 \mathrm{~kg}$, los cuales difieren de los valores reportados por Vilaboa, Bozzi, Díaz y Bazzi (2010), para las razas Pelibuey, Kathadin y Dorper, con valores de $41.13 \mathrm{~kg}, 52.53 \mathrm{~kg}$ y 57.83 $\mathrm{kg}$, respectivamente en ovejas de 3.4 a 4.2 años. En cambio estos valores se aproximan a los reportados por Moreno, Montes, Ucrós, Fernández y Cardona. (2013) en ovejas de pelo colombianas mayores de 2 años con (PV) promedio de $30.2 \mathrm{~kg}$. Y por lo tanto en base a los señalado por Caravaca et al. (2003), estas se clasificarían como animales elipométricos, al estar por debajo de la media general de la especie que esta entre 40 y $70 \mathrm{~kg}$. 
Medidas cefálicas. El ancho de la cabeza (AC), alcanzo un valor promedio de $9.3 \mathrm{~cm}$, ubicándose entre los 8.16 cm, reportados por Arredondo (2013), en ovejas Peli buey, y los $10.9 \mathrm{~cm}$ reportados por Moreno et al. (2013) en ovejas de pelo colombianas, e inferiores a los reportados por Vilaboa et al. (2010), de $11.3 \mathrm{~cm}, 11.93 \mathrm{~cm}$ y $12.23 \mathrm{~cm}$, para Pelibuey, Kathadin y Dorper respectivamente en edades que fluctuaron entre 3.4 y 4.2 años. El coeficiente de variación con un valor de $9.49 \%$ resulto en un rango de variación medio, e indica una cierta homogeneidad de la característica.

Largo de la cabeza (LC), alcanzo un promedio de $23.27 \mathrm{~cm}$, valor superior al reportado por Arredondo. (2013) de $21.88 \mathrm{~cm}$ en ovejas Peli buey y similar a los 23.2 reportados por Moreno et al. (2013) en ovejas de pelo colombianas y a los reportados por Macedo, Arredondo y Cervantes (2016), de $23.33 \mathrm{~cm}, 23.32 \mathrm{~cm}$ y $24.40 \mathrm{~cm}$ en ovejas Pelibuey, Kathadin y BlackBelly respectivamente. El coeficiente de variación de esta característica resulto de $4.66 \%$, lo cual hace de ella una característica muy uniforme en las ovejas estudiadas.

Largo de la cara (LCA), resulto de $15.08 \mathrm{~cm}$, valor que está por debajo del $20.6 \mathrm{~cm}$ reportado por Moreno et al. (2013), sin embargo, se aproxima más a los valores de $14.5 \mathrm{~cm}, 14.64 \mathrm{~cm}$ y $15.8 \mathrm{~cm}$ reportados por Macedo et al. (2016), para ovejas Pelibuey, Kathadin y BlackBelly, respectivamente. El coeficiente de variación de esta característica resulto bajo con un $4.56 \%$, denotando una buena uniformidad en esta variable.

Longitud cráneo: resulto con una longitud promedio de $8.20 \mathrm{~cm}$, valor que está por encima al reportado por Arredondo. (2013) de $6.53 \mathrm{~cm}$ en ovejas Peli buey y muy próximo a los presentados por Macedo et al. (2016), para ovejas Pelibuey, Kathadin y BlackBelly, con valores respectivos de $8.83 \mathrm{~cm}, 8.68 \mathrm{~cm}$ y $8.60 \mathrm{~cm}$. El coeficiente de variación de la característica registró un valor de 8.48 , con lo cual se puede considerar de variación media, es decir de mediana homogeneidad.

La escasa variabilidad encontrada en las medidas cefálicas, puede estar relacionada con la poca influencia que estas tienen de parte de los factores ambientales o por el manejo que reciben los animales (Herrera y Luke, 2009), por lo tanto, el manejo y alimentación que estas ovejas reciban, no será un factor influyente en la variación de estas medidas.

Medidas del tronco y extremidades. La Alzada a la cruz (ALC), resulto de $63.53 \mathrm{~cm}$, magnitud inferior a las reportadas por Arredondo. (2013) de $64.92 \mathrm{~cm}$, en ovejas Pelibuey y los $65.18 \mathrm{~cm}, 67.14 \mathrm{~cm}$ y $65.14 \mathrm{~cm}$, reportados por Vilaboa et al. (2010), para ovejas Pelibuey, Kathadin y Dorper, respectivamente y también al reportado por Moreno et al. (2013) de $64.6 \mathrm{~cm}$ en ovejas de pelo Colombianas. El coeficiente de variación resulto de $3.65 \%$ lo cual expresa una buena homogeneidad para dicha característica.

Alzada a la grupa (ALG), esta expreso un valor medio de $62.54 \mathrm{~cm}$, que aproxima a los valores reportados por Moreno et al. (2013), de 63.5 en ovejas de pelo Colombianas y a los reportados por Vilaboa et al. (2010), para ovejas Pelibuey, Kathadin y Dorper, con magnitudes de $64.55 \mathrm{~cm}, 66.62 \mathrm{~cm}$, y $64.98 \mathrm{~cm}$. El coeficiente de variación de la característica alcanzo un valor de $3.79 \%$ que expresa la escaza variabilidad de la misma.

Las alzadas a la cruz asociada a la alzada a la grupa presentan una gran importancia, cuando ambas tienen un mismo valor, el individuo presenta una línea dorso-lumbar recta, favoreciendo la corrección del ángulo de la línea de la grupa, la inserción de los ligamentos de la mama y permite un mayor desarrollo muscular de la región, (Herrera y Luke. 2009). En las ovejas de nuestro estudio observamos que la diferencia entre ambas es de $0.99 \mathrm{~cm}$, lo cual no es una diferencia tan marcada teniendo esta región una tendencia hacia la horizontalidad.

Diámetro longitudinal (DL), alcanzo un valor promedio de $65.53 \mathrm{~cm}$, ligeramente mayor a la longitud corporal de las Pelibuey de $64.05 \mathrm{~cm}$, señalada por Vilaboa et al. (2010), con diferencias en relación a las longitudes registradas por el mismo autor para las razas Kathadin y Dorper con valores de $73.52 \mathrm{~cm}$ y $74.52 \mathrm{~cm}$ respectivamente. Arredondo. (2013), también difiere con la longitud de las ovejas de nuestro estudio, pues reporta largos de cuerpo en Pelibuey de $68.53 \mathrm{~cm}$, sin embargo, todas estas mediadas resultan inferiores a la señalada por Moreno et al. (2013), quien reporto $88.7 \mathrm{~cm}$ para ovejas de pelo colombianas.

Si asociamos los valores encontrados para alzada a la cruz (ALC), de nuestro estudio que resulto de 63.5 $\mathrm{cm}$ y el largo del cuerpo (DL), es claro que el diámetro longitudinal es mayor que la alzada a la cruz, de manera que los individuos de este rebaño presentan proporciones con tendencia ligeramente alargada. La variabilidad de esta característica resulto media, puesto que presento un coeficiente de variación de $8.05 \%$.

Diámetro dorso esternal (DE), o profundidad de pecho, la media alcanzada fue de $26.18 \mathrm{~cm}$, inferior a la reportada por Arredondo. (2013), de $31.16 \mathrm{~cm}$ en ove- 
jas Pelibuey y a la reportada por Moreno et al. (2013), de $31.5 \mathrm{~cm}$, en ovejas de pelo colombianas. El CV de la característica alcanzo un valor de $6.56 \%$, es decir de homogeneidad media.

Ancho de pecho (AP), alcanzo un valor de $16 \mathrm{~cm}$, similar al $16.98 \mathrm{~cm}$, reportado por Arredondo. (2013), e inferior al $17.9 \mathrm{~cm}$ señalado por Moreno et al. (2013), esta variable es de gran importancia, junto con el alto de pecho para determinar la capacidad pulmonar, que en el caso de las ovejas estudiadas sería inferior a las reportadas por los otros autores. El CV alcanzo $6.64 \%$, por lo que se catalogada de homogeneidad media.

Ancho de la grupa (AG), midió en promedio $15.79 \mathrm{~cm}$, resultando inferior a los $16.33 \mathrm{~cm}$, para Pelibuey, $18.38 \mathrm{~cm}$, para Kathadin y $19.02 \mathrm{~cm}$ en la Dorper, reportados por Vilaboa et al. (2010). Y de la misma manera Arredondo. (2013), y Moreno et al. (2013) reportaron mayores valores de 17.63 y 16.9 respectivamente. El CV, alcanzo 5.8\%, (homogeneidad media).

Largo de la grupa (LG), alcanzo un promedio de $20.3 \mathrm{~cm}$, muy próxima a los valores reportados por Moreno et al. (2013), de $19 \mathrm{~cm}, \mathrm{y}$ a los $20.61 \mathrm{~cm}$ señalados por Arredondo. (2013). De igual proximidad, resultaron los reportes de Vilaboa et al. (2010), con magnitudes de 19.29 cm, 21.56 y 21.65, para Pelibuey, Kathadin y Dorper respectivamente. La longitud y anchura de la grupa, reviste gran importancia en una raza, ya que la estructura que esta tiene, se relaciona con la aptitud reproductiva, específicamente con la facilidad de parto. Por otro lado, esta región es de gran importancia por ser asiento de grandes paquetes musculares que a su vez están catalogados como carne de buena calidad, la amplitud de esta región condiciona las características del muslo y de la pierna (Mella, 2010). El CV resulto de $16.76 \%$, que cataloga la característica de muy heterogénea.

Perímetro torácico (PT), alcanzo un valor de $83.57 \mathrm{~cm}$, similar al reportado por Vilaboa et al. (2010) de $81.2 \mathrm{~cm}$ para Pelibuey y 84.85 en Kathadin, reportando el mismo autor un valor más alto para las Dorper con $87.78 \mathrm{~cm}$. Arredondo. (2013) y Moreno et al. (2013), reportaron valores inferiores de $77.24 \mathrm{~cm}$ en Pelibuey mexicana y $71.9 \mathrm{~cm}$ en la criolla colombiana, respectivamente. El coeficiente de variación encontrado en esta variable alcanzo un $4.83 \%$, lo cual da una idea de un buen nivel de homogeneidad.

Perímetro de la caña (PC), este alcanzo un valor medio de $12.11 \mathrm{~cm}$, que está por encima de los reportados por Vilaboa et al. (2010), de $8.74 \mathrm{~cm}, 9.69 \mathrm{~cm}$ y 9.82 cm, para Pelibuey, Kathadin y Dorper respectivamente. Otros valores inferiores también fueron señalados por Arredondo. (2013) en Pelibuey mexicana con $7.32 \mathrm{~cm}$ y Moreno et al. (2013), en ovejas de pelo Colombianas con $7.1 \mathrm{~cm}$. Herrera y Luque. (2009), señalan que esta medida tiene un valor diferenciador entre las razas destinadas a la producción de carne (cañas medianas a grandes) y de leche (cañas de mediano grosor a finas). La variabilidad de la característica resulto intermedia con un CV de $6.74 \%$.

Índices etnológicos. El índice cefálico con un valor de 42.66 clasifica a estos animales, como dolicocéfalos al predominar el largo de la cabeza sobre el ancho. Autores como Arredondo. (2013), reporta un valor de 39.44 para dicho índice en ovejas Pelibuey en colima México, y Moreno et al. (2013), señala 43.5 para hembras criollas de pelo en Colombia. Herrera y Luque. (2009), señalan que, si los caracteres étnicos suministrados por la cabeza tienen su importancia etnológica, es sobre todo porque su somación no está influenciada por los factores ambientales y por el manejo; y porque su estudio resulta además de mucho más interés en cuanto que son escasos los trabajos biométricos en esta zona corporal.

El índice dáctilo torácico (IDT), alcanzo un valor de 14.54 , valor superior a 11 y por lo tanto de clasificación hipermétrica. Moreno et al. (2013), señala 9.9 para hembras criollas colombianas y Arredondo. (2013), 9.52 en hembras Pelibuey en Colima, por lo que el valor encontrado en nuestro estudio resulta mayor que en ambos casos.

El índice corporal (ICo), alcanzo en promedio un valor de 75.94, con lo cual y de acuerdo a Aparicio (Citado por Vilaboa y Díaz, 2006), se trata de animales brevilíneos, (brevi< ó $=85$, meso entre 86 y 88 y longilíneos $>$ ó $=90)$. En las Peli buey de colima, Arredondo. (2013), reporto un valor de 89.13 y Moreno et al. (2013), un valor de 82 en las criollas colombianas, resultando en todo caso valores superiores al encontrado en las ovejas de nuestro estudio.

Índice de proporcionalidad IPRO alcanzo un valor de 99.84, con lo cual estos animales se clasifican como mediolíneos, y de poca actitud cárnica, ya que de acuerdo con Parés (2009), a menor valor (predominio de la longitud corporal sobre la alzada a la cruz), la forma del animal se aproxima más a un rectángulo, forma característica de los animales de aptitud carnicera. Arredondo. (2013), encontró valores para dicho índice de 95.10 en hembras Pelibuey de Colima y asegura que estos ovinos mostraron una forma casi cuadrangular, característica de los animales de aptitud 
lechera. Moreno et al. (2013), en las criollas colombianas reporta 111.6, que resulta un valor muy superior a los señalados.

El índice pelviano (IPE), según los valores que alcance, puede someterse a una clasificación que señala como convexilínea para un (IPE) $<100$, horizontal (IPE) $=100$ o concavilínea (IPE) $>100$, y en todo caso nos dará una idea de las proporciones de la grupa. El valor que alcanzaron nuestras ovejas fue de 79.49 , lo que permite afirmar que se trata de animales convexilíneos, predominando el largo de la grupa sobre su ancho. Arredondo. (2013), reporto 87.47 en hembras Pelibuey de Colima y en criollas colombianas, Moreno et al. (2013), señala un índice de 75.9, coincidiendo ambos en la clasificación convexilínea.

Cuadro 2. Índices zoométricos de las de ovejas en estudio

\begin{tabular}{|c|c|c|c|c|c|}
\hline Variables & Media & Dev St & $\mathrm{CV}$ & Mínimo & Máximo \\
\hline \multicolumn{6}{|l|}{ Índices etnológicos } \\
\hline Índice cefálico (IC) & 42.66 & 3.27 & 7.70 & 28.17 & 45.88 \\
\hline Índice dáctilo Torácico (IDT) & 14.54 & 1.47 & 10.17 & 12.50 & 19.18 \\
\hline Índice corporal. (ICo) & 75.94 & 3.66 & 4.83 & 73.17 & 86.36 \\
\hline I. de proporcionalidad (IPRO) & 99.84 & 4.76 & 4.77 & 93.22 & 113.12 \\
\hline Índice Pelviano (IPE) & 79.49 & 12.12 & 15.24 & 48.18 & 104.66 \\
\hline \multicolumn{6}{|l|}{ Índices de capacidad productiva } \\
\hline I. prof. relativa del tórax (IPRT) & 41.17 & 1.79 & 4.35 & 37.20 & 45.39 \\
\hline I. Pelviano transversal. (IPET) & 24.85 & 1.18 & 4.79 & 21.77 & 26.47 \\
\hline I. Pelviano longitudinal (IPEL) & 31.96 & 5.29 & 16.56 & 24.42 & 52.05 \\
\hline $\begin{array}{l}\text { I. Espesor relativo de caña. } \\
\text { (IERC) }\end{array}$ & 19.10 & 1.65 & 8.66 & 16.17 & 23.89 \\
\hline
\end{tabular}

Índice de capacidades productivas o funcionales. El índice de profundidad relativa del tórax (IPRT), alcanzo un valor de 41.17 , por debajo de 50 y por lo tanto con poca orientación carnicera. Arredondo. (2013), coincidió con nuestro estudio al reportaron índice de 48.05 en hembras Pelibuey de Colima, y definiéndolas en base a ese valor como de baja orientación carnicera.

El índice de espesor relativo de la caña (IERC), muestra la relación entre el perímetro del hueso de la caña y la altura del animal, siendo mayor en razas con aptitud de carne, en nuestro caso presentó un valor de 19.10, muy superior, al índice de 11 reportado por Moreno et al. (2013), en las ovejas de pelo colombiana y al 11.32, reportado por Arredondo. (2013), en las ovejas Peli buey de colima.

Las variables fanerópticas observadas. En el cuadro 3 , puede observarse como los colores predominantes de las capas son, café y Negra seguida por las tonalidades donde intervienen varios colores, alcanzando las menores proporciones las amarillentas y blancas. Montes et al. (2013), reporto en ovejas de pelo colombianas predominio de capas cafés, seguida por las amarillentas, y en menores proporciones las blancas, negras y con la intervención de varios colores, predominando en estas ovejas los colores cafés y amarillenta.

En relación a la coloración de las mucosas nasales, predomino la pigmentación negra, seguida de la coloración rosada y en baja proporción las despigmentadas. En ovejas de pelo colombianas, Montes et al. (2013), coincide al encontrar un mayor número de ovejas con mucosas negras, pero difiere en las siguientes que fueron las oscurecidas y de manera mínima las rosadas.

En la pigmentación de las pezuñas destaco la tonalidad oscura, seguida por las tonalidades claras y en mínima proporción las tonalidades manchadas. Montes et al. (2013), en su estudio difiere al reportar como pigmentación predominante el castaño claro (carmelita), seguida de los tonos oscuros y en menor cuantía las pezuñas de tonos claros.

Cuadro 3. Frecuencia de las características fanerópticas

\begin{tabular}{clcc}
\hline Carácter & Indicador & $\begin{array}{c}\text { Valor } \\
\text { absoluto }\end{array}$ & $\begin{array}{c}\text { Frecuencia } \\
\text { relativa (\%) }\end{array}$ \\
\hline \multirow{4}{*}{ Color de la capa } & Café & 8 & 25.80 \\
& Amarillenta & 5 & 16.12 \\
& Blanca & 3 & 9.67 \\
& Negra & 8 & 25.80 \\
& Color mezclado & 7 & 22.58 \\
Color de las mucosas & Negras & 22 & 71.00 \\
& Rosadas & 7 & 23.00 \\
& Despigmentadas & 2 & 6.00 \\
Pigmentación de pezuñan & Claras & 10 & 65.00 \\
Pigmentación de ubre & Obscuras & 1 & 32.00 \\
& Pigmentada & 20 & 3.00 \\
Inclinación de la grupa & Despigmentada & 8 & 74.00 \\
& Recta & 26 & 26.00 \\
Perfil cefálico & Inclinada & 5 & 84.00 \\
& Semi-convexo & 16 & 16.00 \\
& Convexo & 2 & 52.00 \\
& Recto & 13 & 6.00 \\
Dirección de las orejas & Caídas & 9 & 42.00 \\
& Horizontales & 22 & 71.00 \\
\hline
\end{tabular}

Fue claro que las ubres pigmentadas, prevalecieron sobre las no pigmentadas, lo que difiere con lo reportado para las ovejas de pelo colombianas, donde destaca de manera muy clara el predominio de las ubres despigmentadas y pocos individuos con pigmentación a nivel de la glándula mamaria. 
Las ovejas de nuestro estudio se caracterizaron por presentar mayor proporción de ubres rectas y menos de posición inclinada. Tales resultados pueden estar influenciados, por el hecho que todas las razas que han influido en el hato, presentan grupas rectas (Pelibuey, Blackbelly, Kathadin y Dorper). Montes et al. (2013), de manera contraria encontró en su estudio el predominio de grupas inclinadas y un mínimo de horizontales.

En el perfil de las ovejas, predomino el tipo ligeramente convexo, en el que probablemente influya la característica del perfil de la Pelibuey, seguido del tipo recto, que suponemos este influenciado por las razas Kathadin y Blackbelly, y un mínimo de individuos con perfil convexo, probablemente influenciado por la raza Dorper.

La mayor cantidad de individuos presento orejas con dirección horizontal recta hacia los lados, y en menor proporción los de orejas caídas.

Relación entre las variables corporales estudiadas. En el cuadro 4, pueden observarse todas las correlaciones entre las diferentes variables, y es notorio que estas van, desde muy bajas 0.034, entre largo de la grupa (LG) y ancho de la grupa (AG), hasta muy altas 0.991, entre alzada a la grupa (ALG) y alzada a la cruz (ALC). La mayor parte de las correlaciones entre las variables estudiadas resultaron positivas, con la excepción de largo de la grupa (LG) que se correlaciona negativamente con las variables, peso vivo (PV), y ancho de pecho (AP), y es bien notorio como la variable perímetro de la caña (PC) se correlaciona negativamente con todas las variables estudiadas. En relación a las significancias estadísticas de las correlaciones encontradas, la mayoría resultaron significativas, con la excepción de las correlaciones entre ancho de pecho (AP) y ancho de la cabeza (AC), y de manera muy característica, las correlaciones entre las variables, largo de la grupa (LG) y perímetro de la caña (PC), presentaron correlaciones no significativas respecto a todas las demás variables.

Diversos trabajos como los de, Valdez, Mohamed, Amín, y Varade (citados por Moreno et al, 2013), han demostrado que existe una alta correlación entre el peso vivo (PV) y diferentes medidas corporales tales como: perímetro torácico (PT), alzada a la grupa (AG), alzada a la cruz (AC), etc.). Nuestro estudio es coincidente con tal afirmación, puesto que el peso vivo (PV), respecto a las variables, perímetro torácico (PT), alzada a la cruz (ALC) y alzada a la grupa (ALG), resulto con correlaciones altas y significativas de $0.797,0.883$ y 0.886 , respectivamente. A lo que hay que agregar el diámetro longitudinal (DL) que presento una correlación de 0.841 y la edad con un valor de 0.806 respecto a (PV).

En relación a la correlación alta y significativa de 0.806 encontrada en nuestro estudio entre la edad y peso vivo (PV), es importante tener en cuenta lo señalado por Vilaboa y Díaz, (2006), quienes aseguran que a mayor edad en los ovinos se pierde peso. Y por otro lado los mismos autores también coinciden con nuestro resultado en la correlación positiva que existe entre el diámetro longitudinal (DL) y el peso vivo (PV).

Cuadro 4. Matriz de correlaciones entre todas las medidas biométricas. $P<0.050 \quad n=31$

\begin{tabular}{|c|c|c|c|c|c|c|c|c|c|c|c|c|c|c|}
\hline VAR & PV & $\mathrm{AC}$ & LC & LCA & LCR & ALC & ALG & DL & $\mathrm{AP}$ & $\mathrm{AG}$ & LG & $\mathrm{DE}$ & $\mathrm{PC}$ & PT \\
\hline $\mathrm{AC}$ & 0.701 & & & & & & & & & & & & & \\
\hline$<0.050$ & 0.000 & & & & & & & & & & & & & \\
\hline$<0.050$ & 0.000 & 0.002 & & & & & & & & & & & & \\
\hline LCA & 0.782 & 0.544 & 0.835 & & & & & & & & & & & \\
\hline LCR & 0.727 & 0.721 & 0.611 & 0.661 & & & & & & & & & & \\
\hline$<0.050$ & 0.000 & 0.000 & 0.001 & 0.000 & & & & & & & & & & \\
\hline ALC & 0.883 & 0.702 & 0.850 & 0.917 & 0.764 & & & & & & & & & \\
\hline$<0.050$ & 0.000 & 0.000 & 0.000 & 0.000 & 0.000 & & & & & & & & & \\
\hline
\end{tabular}


Cuadro 4. Continuación...

\begin{tabular}{|c|c|c|c|c|c|c|c|c|c|c|c|c|c|c|}
\hline $\mathrm{DL}$ & 0.841 & 0.559 & 0.799 & 0.822 & 0.600 & 0.924 & 0.906 & & & & & & & \\
\hline$<0.050$ & 0.000 & 0.003 & 0.000 & 0.000 & 0.001 & 0.000 & 0.000 & & & & & & & \\
\hline $\mathrm{AP}$ & 0.515 & 0.379 & 0.505 & 0.502 & 0.512 & 0.558 & 0.568 & 0.599 & & & & & & \\
\hline$<0.050$ & 0.007 & 0.056 & 0.009 & 0.009 & 0.008 & 0.003 & 0.002 & 0.001 & & & & & & \\
\hline $\mathrm{AG}$ & 0.626 & 0.428 & 0.531 & 0.558 & 0.495 & 0.580 & 0.589 & 0.551 & 0.806 & & & & & \\
\hline$<0.050$ & 0.000 & 0.000 & 0.000 & 0.000 & 0.000 & 0.000 & 0.000 & 0.000 & 0.000 & & & & & \\
\hline LG & -0.002 & 0.232 & 0.075 & 0.118 & 0.137 & 0.181 & 0.172 & 0.118 & -0.033 & 0.034 & & & & \\
\hline$<0.050$ & 0.993 & 0.255 & 0.715 & 0.566 & 0.506 & 0.376 & 0.401 & 0.567 & 0.872 & 0.869 & & & & \\
\hline $\mathrm{DE}$ & 0.621 & 0.529 & 0.595 & 0.711 & 0.546 & 0.795 & 0.802 & 0.761 & 0.615 & 0.548 & -0.060 & & & \\
\hline$<0.050$ & 0.001 & 0.006 & 0.001 & 0.000 & 0.004 & 0.000 & 0.000 & 0.000 & 0.001 & 0.004 & 0.771 & & & \\
\hline $\mathrm{PC}$ & -0.158 & -0.141 & -0.304 & -0.223 & -0.157 & -0.279 & -0.282 & -0.295 & -0.180 & -0.015 & -0.157 & -0.290 & & \\
\hline$<0.050$ & 0.411 & 0.492 & 0.131 & 0.273 & 0.445 & 0.167 & 0.163 & 0.143 & 0.379 & 0.943 & 0.443 & 0.151 & & \\
\hline PT & 0.797 & 0.681 & 0.763 & 0.863 & 0.729 & 0.919 & 0.938 & 0.811 & 0.495 & 0.505 & 0.173 & 0.858 & -0.346 & \\
\hline$<0.050$ & 0.000 & 0.000 & 0.000 & 0.000 & 0.000 & 0.000 & 0.000 & 0.000 & 0.010 & 0.009 & 0.399 & 0.000 & 0.083 & \\
\hline EDAD & 0.806 & 0.655 & 0.765 & 0.887 & 0.719 & 0.944 & 0.951 & 0.857 & 0.484 & 0.477 & 0.183 & 0.858 & -0.366 & 0.989 \\
\hline$<0.050$ & 0.000 & 0.000 & 0.000 & 0.000 & 0.000 & 0.000 & 0.000 & 0.000 & 0.012 & 0.014 & 0.370 & 0.000 & 0.066 & 0.000 \\
\hline
\end{tabular}

Análisis multivariado de componentes principales. Se realizó un análisis de componentes principales para determinar las medidas corporales que más contribuyen con la explicación de la varianza. En el cuadro 5, aparecen los resultados del ACP (auto valores -eigenvalues- de cada componente principal, proporción de varianza explicada por cada una de ellos, y varianza explicada acumulada).

Cuadro 5. Valores de componentes principales a partir de la matriz de correlaciones.

\begin{tabular}{cccc}
\hline $\mathrm{CP}$ & $(\lambda \mathrm{i})$ & \% VCP & \% VCP (acumulada) \\
\hline $\mathrm{CP} 1$ & 8.08 & 0.578 & 0.578 \\
$\mathrm{CP} 2$ & 2.27 & 0.162 & 0.740 \\
$\mathrm{CP} 3$ & 1.31 & 0.094 & 0.833 \\
$\mathrm{CP} 4$ & 0.62 & 0.045 & 0.878 \\
$\mathrm{CP} 5$ & 0.42 & 0.030 & 0.908 \\
$\mathrm{CP} 6$ & 0.35 & 0.026 & 0.934 \\
$\mathrm{CP} 7$ & 0.25 & 0.018 & 0.952 \\
$\mathrm{CP} 8$ & 0.20 & 0.015 & 0.966 \\
$\mathrm{CP} 9$ & 0.14 & 0.011 & 0.977 \\
$\mathrm{CP} 10$ & 0.13 & 0.009 & 0.986 \\
$\mathrm{CP} 11$ & 0.10 & 0.008 & 0.994 \\
$\mathrm{CP} 12$ & 0.05 & 0.004 & 0.998 \\
$\mathrm{CP} 13$ & 0.03 & 0.002 & 1.000 \\
$\mathrm{CP} 14$ & -0.00 & -0.00 & 1.000
\end{tabular}

$\mathrm{CP}$ : componente principal, Auto valores $(\lambda \mathrm{i})$ y porcentaje de la varianza explicada por los componentes $(\% \mathrm{VCP})$ para caracteres morfológicos de ovejas.
Los primeros tres componentes tienen todos varianzas (auto valores) mayores que 1 y entre los tres recogen el $83 \%$ de la varianza total. De todos los componentes principales, once $(78.57 \%)$ presentaron varianza menor que 0.7 (auto valor inferior a 0.7 ), por lo que se descartarían de acuerdo al criterio de Jollife (1972, 1973), citado por Carril et al. (2011).

En el cuadro 6, se presentan los 3 componentes que recogen en total el $83 \%$ de la varianza total. Puede observarse como el primer componente principal, se caracteriza por correlaciones positivas con todos los rasgos, con la excepción de perímetro de la caña (PC), siendo la variable de mayor peso el diámetro dorso esternal $\mathrm{DE}$ (0.30), las demás variables con carga relevante positiva son: alzada a la grupa ALG (0.295), ancho de la grupa AG (0.294), alzada a la cruz y diámetro longitudinal ALC y DL (0.292), largo del cráneo LCR (0.286) y largo de la cara LCA (0.283), este primer componente principal recogió el $57.8 \%$ de la variación en las variables originales.

En el segundo componente principal, se encontró un $50 \%$ de correlaciones positivas y la misma proporción de negativas entre los rasgos. De las variables positivas las de mayor relevancia fueron ancho de la cabeza AC y largo de la cabeza LC (0.396) y peso vivo PV (0.395). Las variables con carga negativa fueron ALC, DL, AP, LG, AG, DE y PT, explicando este componente un $16.2 \%$ de la variación total. 
El tercer componente principal, da el mayor peso positivo a ancho de la grupa AG (0.375), y como variable de mayor peso relevante a: perímetro torácico PT (0.353), diámetro dorso esternal DE $(0.297)$ y peso vivo PV (0.257), representando este componente el $9.4 \%$ de la variación total.

Cuadro 6. Autovectores correspondientes a los factores seleccionados

\begin{tabular}{llll}
\hline Variables & CP1 & CP2 & CP3 \\
\hline PV & 0.199 & 0.395 & 0.257 \\
AC & 0.234 & 0.396 & 0.195 \\
LC & 0.251 & 0.396 & 0.041 \\
LCA & 0.283 & 0.301 & -0.099 \\
LCR & 0.286 & 0.215 & -0.193 \\
ALC & 0.292 & 0.077 & -0.279 \\
ALG & 0.295 & -0.111 & -0.301 \\
DL & 0.292 & -0.219 & -0.265 \\
AP & 0.245 & -0.153 & -0.381 \\
LG & 0.267 & -0.279 & -0.133 \\
AG & 0.294 & -0.088 & 0.375 \\
DE & 0.302 & -0.221 & 0.297 \\
PC & -0.273 & 0.306 & -0.303 \\
PT & 0.200 & -0.275 & 0.353 \\
\hline
\end{tabular}

\section{CONCLUSIONES}

Los índices zoométricos determinados, indican que se trata ovinos, dolicocéfalos, hipermétricos, brevilíneos, mediolíneos y convexilíneos.

Los índices de proporcionalidad (IPRO), profundidad relativa del tórax (IPRT) y el pelviano transversal (IPET), revelaron que estos animales, no presentan un formato con clara definición a la actitud cárnica.

Existe predominio de las capas café y negras, mucosas negras, pezuñas oscuras, ubres pigmentadas, grupas rectas, perfiles ligeramente convexos y orejas horizontales hacia los lados.

La correlación más alta encontrada fue entre ALC y ALG, y únicamente, perímetro de la caña (PC), presento disociación absoluta con todas las demás variables, siendo la mayoría de dichas correlaciones positivas y significativas, a excepción de la relación entre (LG) y (PC).

El ACP de las 14 variables utilizadas, revelo que los 3 primeros CP explicaron el $83 \%$ de la variación. Por lo tanto, la información de estos $\mathrm{CP}$, principalmente los valores de los coeficientes, permiten seleccionar 6 variables que revelan el mayor peso: $\mathrm{LC}, \mathrm{AC}, \mathrm{PV}, \mathrm{LG}, \mathrm{PT}$ y DE, que potencialmente pudiesen tenerse en cuenta para la creación de índices de selección.

\section{REFERENCIAS BIBLIOGRÁFICAS}

Alencastre, R. (1991). Producción de ovinos. Universidad Nacional del Altiplano UNA, Puno Perú.

Bravo, S., y Sepúlveda, N. (2010). Índices Zoométricos en Ovejas Criollas Araucanas. Universidad de la Frontera, Temuco, Chile.

Cruz, P. (2005). Tamaño de muestra agropecuaria. IIESCA. Universidad Veracruzana, México.

Delgado, B, J., y Nogales, S. (2009). Biodiversidad Ovina Iberoamericana. Caracterización y uso sustentable. Córdoba. España.

Herrera, M., y Luque, M. (2009). Morfoestructura y sistemas para el futuro en la valoración morfológica. Valoración morfológica de los animales domésticos. Ministerio de medio ambiente y medio rural y marino. ES. 865 p.

INETER (Instituto Nicaragüense de Estudios Territoriales). (2015). Informes y registros meteorológicos.

Macedo, R., Arredondo, V., y Cervantes, A., (2016). Morfología y la cola de carneros Pelibuey, Katahdin y Blackbelly en Colima, México. Facultad de medicina veterinaria y zootecnia, Universidad de Colima. México. DOI: http//dx.doi.org/10.21753/vmoa.3.3.375

Mella, J. (2010). Evaluación zoométrica de la base materna de la raza ovina Chilota comparada con dos razas ovinas predominantes en las regiones de Los Lagos y Los Ríos. Tesis, para optar al grado de Licenciado en Agronomía. Universidad Austral de Chile. Facultad de Ciencias Agrarias. Escuela de Agronomía.

Montes, D., Moreno, J., Hurtado, N., Ramírez, R., Celis, A., y Garay, G. (2013). Caracterización Faneróptica y Morfológica de la hembra ovina de pelo criollo (Camurra), en la subregión sabanas y golfo de Morrosquillo departamento de Sucre. Rev. Colombiana de Ciencia animal. 5(1): 104-115,2013.

Moreno, J., Montes, D., Ucrós, J., Fernández, A., y Cardona, J. (2013). Variabilidad morfoestructural de la hembra ovina de pelo criollo colombiano. Universidad de Sucre, Sincelejo, Colombia, Facultad de Ciencias Agropecuarias.

Parés, C. (2009). Zoometría. En: Valoración Morfológica de los animales domésticos. Sañudo, A.C. Edit. Madrid, Ministerio de Medio Ambiente y Medio Rural y Marino. Recuperado de www.mapa.gob.es/es/ganaderia/temas/zootecnia.

Vilaboa, J., y Díaz, R. (2006). Conformación corporal de las razas ovinas Pelibuey, Dorper y Kathadin, en el estado de Veracruz. MX. Recuperado de https://www.engormix.com/ovinos/articulos/conformacion-corporal-razas-ovinas-t27032.htm

Vilaboa, J., Bozzi, R., Díaz, P., y Bazzi, L. (2010). Conformación corporal de las razas ovinas Peli buey, Dorper y Kathadin, en el estado de Veracruz. MX. Zootecnia Trop., 28(3): 321-328. 2010. Recuperado de ttps://www.researchgate.net/publication/262631742. 